\title{
A Study on Some New Results Arising from $(p, q)$-Calculus
}

\author{
Ugur Duran ${ }^{1, *}$, Mehmet Acikgoz ${ }^{2}$ and Serkan Araci ${ }^{3}$ \\ ${ }^{1}$ Department of Basic Concepts of Engineering, \\ Faculty of Engineering and Natural Science, \\ İskenderun Technical University, TR-31200 Hatay, Turkey \\ E-Mail: mtdrnugur@gmail.com \\ *Corresponding Author \\ ${ }^{2}$ Department of Mathematics, Faculty of Arts and Science, \\ University of Gaziantep, TR-27310 Gaziantep, Turkey \\ E-Mail: acikgoz@gantep.edu.tr \\ ${ }^{3}$ Department of Economics, Faculty of Economics, \\ Administrative and Social Science, \\ Hasan Kalyoncu University, TR-27410 Gaziantep, Turkey \\ E-Mail: mtsrkn@hotmail.com
}

\begin{abstract}
This paper includes some new investigations and results for post quantum calculus, denoted by $(p, q)$-calculus. A chain rule for $(p, q)$-derivative is developed. Also, a new $(p, q)$-analogue of the exponential function is introduced and some its properties including the addition property for $(p, q)$-exponential functions are investigated. Several useful results involving $(p, q)$-binomial coefficients and $(p, q)$-antiderivative are discovered. At the final part of this paper, $(p, q)$-analogue of some elementary functions including trigonometric functions and hyperbolic functions are considered and some properties and relations among them are analyzed extensively.
\end{abstract}

2010 Mathematics Subject Classification: 05A30, 33A10.

Key Words and Phrases. $q$-calculus; $(p, q)$-calculus; exponential functions; trigonometric functions; hyperbolic functions.

\section{Introduction}

As $q$-calculus including $q$-number with one base $q$ is dealt with by some scientists, $(p, q)$ calculus including $(p, q)$-number with independent two variables $p$ and $q$ are firstly considered circa the same time (1991) and independently by Chakrabarti and Jagannathan [4], Brodimas et al. [2], Wachs and White [25], and Arik et al. [1]. Chakrabarti and Jagannathan [4] introduced the $(p, q)$-number to generalize or unify several forms of $q$-oscillator algebras well-known in the physics literature related to the representation theory of single parameter quantum algebras. Brodimas et al. [2] introduced $(p, q)$-number so that $(p, q)$-algebra can be derived from $q$-calculus by a Bargmann differential realization of the creation and annihilation operator, the Bose representation of those operators was derived, and $(p, q)$-Harmonic oscillator was constructed. Wachs and White [25] introduced $(p, q)$-number in mathematical literature in order to obtain $(p, q)$-Stirling number which is the generating function the 
joint distribution of pairs of statistics. Arik et al. [1] introduced $(p, q)$-number to investigate Fibonacci oscillators. Thereby, several physical and mathematical problems lead to the necessity of $(p, q)$-calculus. Based on the aforementioned papers, many mathematicians and physicists have developed the $(p, q)$-calculus in many different research areas since 1991. For instance, Burban and Klimyk [3] introduced $(p, q)$-hypergeometric functions and studied the relations among the basic hypergeometric functions, $q$-hypergeometric functions, and $(p, q)$-hypergeometric functions in 1994. Jagannathan [16] considered a more general $(p, q)$ hypergeometric series as well as Burban's $(p, q)$-hypergeometric series in [3] and he derived some related preliminary results in 1997. In 2005, Jagannathan and Srinivasa [15] gave a method to embed the $q$-series into a $(p, q)$-series, discovered some results corresponding $(p, q)$-extensions of the known $q$-identities and they also studied on $(p, q)$-hypergeometric series, based on the $(p, q)$-numbers. In 2008, Corcino [7] developed the $(p, q)$-extension of the binomial coefficients and also he established some properties parallel to those of the ordinary and $q$-binomial coefficients. Sadjang [22] investigated some properties of the $(p, q)$ derivative and the $(p, q)$-integration and presented two appropriate polynomials basis for the $(p, q)$-derivative, and then he derived various properties of these bases in the year 2013. As an application, he gave two $(p, q)$-Taylor formulas for polynomials. Furthermore, the fundamental theorem of $(p, q)$-calculus and the formula of $(p, q)$-integration by part were given. In the year 2015, Sadjang [23] introduced a new generalization of the Gamma and the Beta functions, calling as $(p, q)$-Gamma and $(p, q)$-Beta functions and developed some their properties which reduce to the known results as special cases. Mursaleen et al. ([19], [20]) introduced a new analogue of Bernstein operators, calling as $(p, q)$-Bernstein operators that are a generalization of $q$-Bernstein operators and they also considered approximation properties based on Korovkin's type approximation theorem of $(p, q)$-Bernstein operators, and they established some direct theorems in 2015. The $(p, q)$-analogues of Bernoulli polynomials, Euler polynomials, and Genocchi polynomials were described by Duran et al. [9] in early 2016 and the $(p, q)$-analogues of known earlier formulae were obtained, and relations between the new and old polynomials were investigated by making use of the fermionic $p$-adic integral over the $p$-adic number fields. Also in early 2016, a new class of Bernoulli, Euler and Genocchi polynomials based on the theory of $(p, q)$-calculus were considered by Duran et al. [12] and their some properties including addition theorems, difference equations, derivative properties, recurrence relationships were investigated. The $(p, q)$-extension of Cheon's main result in [5] was acquired, and further, the $(p, q)$-analogue of the main results given earlier by Srivastava and Pintér in [24] was discovered in [12]. Duran et al. [11] researched and gave some connections between the $(p, q)$-derivative operator and divided differences in 2016. Moreover, in 2016, Duran et al. [8] considered an extension of Haar distribution based on $(p, q)$-numbers. By means of this distribution, the $(p, q)$-analogue of Volkenborn integration that is a new generalization of $q$-Volkenborn integration was derived. Some properties of Volkenborn integration based on $(p, q)$-numbers were investigated. Finally, $(p, q)$-Bernoulli numbers and polynomials derived from $(p, q)$-Volkenborn integral were constructed and also some their properties were obtained.

We now review briefly some concepts of $(p, q)$-calculus. 
We begin with the following notations: $\mathbb{N}$ denotes the set of the natural numbers, $\mathbb{N}_{0}$ denotes the set of nonnegative integers, $\mathbb{R}$ denotes the set of real numbers and $\mathbb{C}$ denotes the set of complex numbers.

The twin-basic number or $(p, q)$-number is defined by, for any number $n$,

$$
[n]_{p, q}:=\frac{p^{n}-q^{n}}{p-q}=p^{n-1}+p^{n-2} q+\cdots+p q^{n-2}+q^{n-1}
$$

which is a natural generalization of the $q$-number such that

$$
\left.[n]_{p, q}\right|_{p=1}:=[n]_{q}=\frac{1-q^{n}}{1-q}=1+q+\cdots+q^{n-2}+q^{n-1} .
$$

Note that $[n]_{p, q}=[n]_{q, p}$.

The $(p, q)$-derivative of a function $f$ with respect to $x$ is defined by

$$
D_{p, q ; x} f(x):=D_{p, q} f(x)=\frac{f(p x)-f(q x)}{(p-q) x} \quad(x \neq 0)
$$

and $\left(D_{p, q} f\right)(0)=f^{\prime}(0)$, provided that $f$ is differentiable at 0 . As with the $q$-derivative and the ordinary derivative, the action of applying the $(p, q)$-derivative of any function is a linear operator, viz., for any constants $a$ and $b$,

$$
D_{p, q}(a f(x)+b g(x))=a D_{p, q} f(x)+b D_{p, q} g(x) .
$$

The $(p, q)$-derivatives of product and the quotient of $f(x)$ and $g(x)$ are given by

$$
\begin{aligned}
D_{p, q}(f(x) g(x)) & =g(p x) D_{p, q} f(x)+f(q x) D_{p, q} g(x) \\
& =f(p x) D_{p, q} g(x)+g(q x) D_{p, q} f(x)
\end{aligned}
$$

and

$$
\begin{aligned}
D_{p, q}\left(\frac{f(x)}{g(x)}\right) & =\frac{g(q x) D_{p, q} f(x)-f(q x) D_{p, q} g(x)}{g(p x) g(q x)} \\
& =\frac{g(p x) D_{p, q} f(x)-f(p x) D_{p, q} g(x)}{g(p x) g(q x)} .
\end{aligned}
$$

As well as the formulas (1.4) and (1.5) we may write one more representation in symmetrical form

$$
D_{p, q}\left(\frac{f(x)}{g(x)}\right)=\frac{1}{2} \frac{D_{p, q} f(x)(g(p x)+g(q x))-D_{p, q} g(x)(f(p x)+f(q x))}{g(p x) g(q x)} .
$$

The formulas in Eqs. (1.4), (1.5) and (1.6) are valid, however one of this forms may be more useful than others under special cases.

The $(p, q)$-Gauss Binomial formula is defined by

$$
\begin{aligned}
(x \oplus a)_{p, q}^{n} & =\left\{\begin{array}{cl}
(x+a)(p x+a q) \cdots\left(p^{n-2} x+a q^{n-2}\right)\left(p^{n-1} x+a q^{n-1}\right), & \text { if } n \geq 1 \\
1, & \text { if } n=0
\end{array}\right. \\
& =\sum_{k=0}^{n}\left[\begin{array}{l}
n \\
k
\end{array}\right]_{p, q} p\left(\begin{array}{c}
k \\
2
\end{array}\right) q^{\left(\begin{array}{c}
n-k \\
2
\end{array}\right)} x^{k} a^{n-k}
\end{aligned}
$$


where the notations $\left[\begin{array}{l}n \\ k\end{array}\right]_{p, q}((p, q)$-Gauss Binomial coefficients $)$ and $[n]_{p, q} !((p, q)$-factorial) are defined by

and

$$
\left[\begin{array}{l}
n \\
k
\end{array}\right]_{p, q}=\frac{[n]_{p, q} !}{[n-k]_{p, q} ![k]_{p, q} !} \quad(n \geq k)
$$

$$
[n]_{p, q} !=[n]_{p, q}[n-1]_{p, q} \cdots[2]_{p, q}[1]_{p, q} \quad(n \in \mathbb{N}) .
$$

The $(p, q)$-exponential functions, $e_{p, q}(x)$ and $E_{p, q}(x)$, are defined by

which hold the basic identity

$$
e_{p, q}(x)=\sum_{n=0}^{\infty} p\left(\begin{array}{l}
n \\
2
\end{array}\right) \frac{x^{n}}{[n]_{p, q} !}
$$

and

The following $(p, q)$-derivatives hold true:

$$
E_{p, q}(x)=\sum_{n=0}^{\infty} q\left(\begin{array}{l}
n \\
2
\end{array}\right) \frac{x^{n}}{[n]_{p, q} !},
$$

$$
D_{p, q} e_{p, q}(x)=e_{p, q}(p x) \text { and } D_{p, q} E_{p, q}(x)=E_{p, q}(q x) .
$$

Note that the $(p, q)$-derivatives of the $(p, q)$-exponentials are not precisely themself. However those derivatives are in the similar form of the derivative of classical exponential functions.

A more detailed statement of above, including $(p, q)$-numbers, is found in $[1-4,7-9,11,12$, $15,16,19,20,22,23,25]$.

Taking here $p=1$, then all notations given in this part reduce to the notations of the usual $q$-calculus (for details see $[6,10,14,17,18,21]$ ).

\section{Main Results}

The formulas (1.2) and (1.3) can be rewritten in explicitly symmetrical form:

$$
D_{p, q}(f(x) g(x))=D_{p, q} f(x)\left(\frac{g(p x)+g(q x)}{2}\right)+D_{p, q} g(x)\left(\frac{f(p x)+f(q x)}{2}\right) .
$$

More general form of the multiplication rule of $(p, q)$-derivative is presented with fixed $0 \leq$ $\alpha \leq 1$

$D_{p, q}(f(x) g(x))=(\alpha f(q x)+(1-\alpha) f(p x)) D_{p, q} g(x)+(\alpha g(p x)+(1-\alpha) g(q x)) D_{p, q} f(x)$.

If we choose $\alpha=1, \alpha=0$ and $\alpha=\frac{1}{2}$, we then get the formulas (1.2), (1.3) and (2.1), respectively.

Let $y=f(x)$ be a injective and surjective mapping. In this case, we have $x=f^{-1}(y)$ where $f^{-1}$ is the inverse function to $f$. Applying $(p, q)$-derivative to each side of $x=f^{-1}(y)$ gives

$$
\begin{aligned}
1 & =D_{p, q} x=D_{p, q} f^{-1}(y) \\
& =\frac{f^{-1}(y(p x))-f^{-1}(y(q x))}{y(p x)-y(q x)} \cdot \frac{y(p x)-y(q x)}{p x-q x} \\
& =D_{p, q ; x} f^{-1}(y(x)) \cdot D_{p, q ; x} y(x),
\end{aligned}
$$


thus we arrive

$$
D_{p, q ; x} f^{-1}(y(x))=\frac{1}{D_{p, q ; x} y},
$$

which is $(p, q)$-extension of the usual derivative of inverse function $f^{-1}$.

As it has been given for $q$-derivative in [17], there doesn't exist a general chain rule for $(p, q)$-derivatives. That is, if we consider the function $f(u(x))$, where $u=u(x)=\lambda x^{\mu}$ with $\lambda, \mu$ being constants, we have a chain rule as special case:

$$
\begin{aligned}
D_{p, q}[f(u(x))] & =D_{p, q}\left[f\left(\lambda x^{\mu}\right)\right]=\frac{f\left(\lambda x^{\mu} p^{\mu}\right)-f\left(\lambda x^{\mu} q^{\mu}\right)}{x(p-q)} \\
& =\frac{f\left(\lambda x^{\mu} p^{\mu}\right)-f\left(\lambda x^{\mu} q^{\mu}\right)}{\lambda x^{\mu} p^{\mu}-\lambda x^{\mu} q^{\mu}} \cdot \frac{\lambda x^{\mu} p^{\mu}-\lambda x^{\mu} q^{\mu}}{x(p-q)} \\
& =\frac{f\left(u p^{\mu}\right)-f\left(u q^{\mu}\right)}{u p^{\mu}-u q^{\mu}} \cdot \frac{u(p x)-u(q x)}{x(p-q)}
\end{aligned}
$$

which gives

$$
D_{p, q} f(u(x))=\left(D_{p^{\mu}, q^{\mu}} f\right)(u(x)) . D_{p, q} u(x)
$$

that is $(p, q)$-extension of the Eq. (1.15) in [17].

Conversely, if we consider the function $u(x)=x^{3}+x^{2}$ or $u(x)=\cos x$, the quantity $u(p x)$ and $u(q x)$ can not be derived in terms of $u$ in a basic way, and thereby it is impossible to write a general chain rule.

As has been done in [21] for multiple $q$-calculus, the case $D_{p, q} \varpi(x)=0$ for any function $\varpi$ if and only if $\varpi(p x)=\varpi(q x)$ called $(p, q)$-periodic function. By choosing $p=e^{\ln p}$ and $x=e^{\ln x}=e^{y}$ we can write $\varpi(x)=\varpi\left(e^{y}\right) \equiv G(y)$ and

$$
\varpi(p x)=G(y+\ln p) .
$$

Using condition of $(p, q)$-periodicity of $F(x)$, we get

$$
G(y+\ln p)=G(y+\ln q)
$$

and if we denote $y+\ln p=z$, then we realize

$$
G(z)=G\left(z+\ln \frac{q}{p}\right)
$$

which implies that $G(z)$ is standart periodic function

$$
G(z)=G(z+t)
$$

with period $t=\ln \frac{q}{p}=\ln q-\ln p$.

Applying $k$ times the derivative operator $D_{p, q}$ to $(a \ominus x)_{p, q}^{n}$ successively yields to the following proportion.

Proposition 1. Let $n$ be a positive integer and $0 \leq k \leq n$, we have

$$
D_{p, q}^{k} \frac{1}{(x \ominus a)_{p, q}^{n}}=(-1)^{k} q\left(\begin{array}{c}
k \\
2
\end{array}\right) \frac{[n+k-1]_{p, q} !}{[n-1]_{p, q} !} \frac{1}{\left(q^{k} x \ominus a\right)_{p, q}^{n+k}} .
$$


In a like manner above, we get the following result.

Proposition 2. Let $n \geq 1$ be an integer and $0 \leq k \leq n$, we have

$$
D_{p, q}^{k} \frac{1}{(a \ominus x)_{p, q}^{n}}=p\left(\begin{array}{c}
k \\
2
\end{array}\right) \frac{[n+k-1]_{p, q} !}{[n-1]_{p, q} !} \frac{1}{\left(a \ominus p^{k} x\right)_{p, q}^{n+k}} .
$$

Now, we analyze some properties of the $(p, q)$-exponential functions. We observe that

$$
e_{\frac{1}{p}, \frac{1}{q}}(x)=\sum_{n=0}^{\infty} \frac{1}{[n]_{\frac{1}{p}, \frac{1}{q} !}} p^{-\left(\begin{array}{c}
n \\
2
\end{array}\right)} x^{n}=\sum_{n=0}^{\infty} \frac{1}{[n]_{p, q} !} q^{\left(\begin{array}{c}
n \\
2
\end{array}\right)} x^{n}
$$

thus, we get

$$
e_{\frac{1}{p}, \frac{1}{q}}(x)=E_{p, q}(x)
$$

In a like manner, we have

$$
E_{\frac{1}{p}, \frac{1}{q}}(x)=e_{p, q}(x) .
$$

How about the additive property of the $(p, q)$-exponential functions? The answer is that the $(p, q)$-exponential functions have not any additive property. Indeed,

$$
\begin{aligned}
e_{p, q}(x) E_{p, q}(y) & =\left(\sum_{n=0}^{\infty} p\left(\begin{array}{l}
n \\
2
\end{array}\right) \frac{x^{n}}{[n]_{p, q} !}\right)\left(\sum_{n=0}^{\infty} q\left(\begin{array}{c}
n \\
2
\end{array}\right) \frac{y^{n}}{[n]_{p, q} !}\right) \\
& \left.=\sum_{n=0}^{\infty}\left(\sum_{k=0}^{n}\left[\begin{array}{l}
n \\
k
\end{array}\right]_{p, q} p\left(\begin{array}{c}
k \\
2
\end{array}\right) q^{(} \begin{array}{c}
n-k \\
2
\end{array}\right) x^{k} y^{n-k}\right) \frac{1}{[n]_{p, q} !} \\
& =\sum_{n=0}^{\infty} \frac{(x \oplus y)_{p, q}^{n}}{[n]_{p, q} !}
\end{aligned}
$$

which is not any form of $(p, q)$-exponential functions $\left(e_{p, q}(x)\right.$ and $\left.E_{p, q}(x)\right)$.

We define a new type $(p, q)$-exponential function as

$$
\widetilde{e}_{p, q}(x)=\sum_{n=0}^{\infty} \frac{x^{n}}{[n]_{p, q} !} .
$$

From Eqs. (2.7) and (2.8), we deduce

$$
e_{p, q}(x) E_{p, q}(y)=\widetilde{e}_{p, q}(x \oplus y)_{p, q},
$$

which can be called addition formula for $(p, q)$-exponentials. Similar to usual exponential function $e^{x}=\sum_{n=0}^{\infty} \frac{x^{n}}{n !}$ and $q$-exponential function $e_{q}(x)=\sum_{n=0}^{\infty} \frac{x^{n}}{[n]_{q} !},(p, q)$-exponential functions has the following derivative property

$$
D_{p, q} \widetilde{e}_{p, q}(x)=\widetilde{e}_{p, q}(x) .
$$

Note that $(p, q)$-Pascal rules are given by $(c f .[7])$

$$
\left[\begin{array}{c}
n+1 \\
k
\end{array}\right]_{p, q}=p^{k}\left[\begin{array}{l}
n \\
k
\end{array}\right]_{p, q}+q^{n-k+1}\left[\begin{array}{c}
n \\
k-1
\end{array}\right]_{p, q}
$$

and

$$
\left[\begin{array}{c}
n+1 \\
k
\end{array}\right]_{p, q}=q^{k}\left[\begin{array}{l}
n \\
k
\end{array}\right]_{p, q}+p^{n-k+1}\left[\begin{array}{c}
n \\
k-1
\end{array}\right]_{p, q} .
$$


Here, we give a theorem about properties of the $(p, q)$-binomial coefficients.

Theorem 1. Each $(p, q)$-binomial coefficient is a polynomial including the parameters $p$ and $q$ of degree $k(n-k)$ whose leading coefficient is 1 .

Proof. This theorem can be proved by the same method in the proof of Corollary 6.1. in $[17]$.

Also, the coefficients in the polynomial expression of $\left[\begin{array}{l}n \\ k\end{array}\right]_{p, q}$ are symmetric.

Note that the $(p, q)$-binomial coefficients also have combinatorial interpretations like $q$ binomial coefficients and usual binomial coefficients.

Definition 1. [22] The function $F(x)$ is a q-antiderivative of $f(x)$ if $D_{p, q} F(x)=f(x)$. It is shown by

$$
\int f(x) d_{p, q} x
$$

The following proposition is a $(p, q)$-generalization of the Proposition 18.1 in the book [17].

Proposition 3. Let $0<q<p \leq 1$. Then, any function $f(x)$ has at most one $(p, q)$ antiderivative which is continuous at $x=0$, up to adding a constant.

Proof. By using the similar proof's technical in [17] for $q$-antiderivative, this theorem can be proved.

Let us consider the following formula for the change of variable $u=u(x)=\lambda x^{\mu}$ with $\lambda, \mu$ being constants. If $F(x)$ is a $(p, q)$-antiderivative of $f(x)$, then we have

$$
\int f(u) d_{p, q} u=F(u)=F(u(x)) .
$$

Using the expression (2.7) we have for any $\widehat{p}$ and $\widehat{q}$

$$
\begin{aligned}
F(u(x)) & =\int D_{\widehat{p}, \widehat{q}} F(u(x)) d_{\widehat{p}, \widehat{q}} x \\
& =\int\left(D_{\widehat{p}^{\mu}, \widehat{q}^{\mu}} F\right)(u(x)) \cdot D_{\widehat{p}, \widehat{q}} u(x) d_{\widehat{p}, \widehat{q}} x \\
& =\int\left(D_{\widehat{p}^{\mu}, \widehat{q}^{\mu}} F\right)(u(x)) d_{\widehat{p}, \widehat{q}} u(x) .
\end{aligned}
$$

If we take $\widehat{p}=p^{1 / \mu}$ and $\widehat{q}=q^{1 / \mu}$, then we have $D_{\widehat{p}^{\mu}, \widehat{q}^{\mu}} F=D_{p, q} F=f$, and hence

$$
\int f(u) d_{p, q} u=\int f(u(x)) d_{p^{1 / \mu}, q^{1 / \mu}} u(x) .
$$

This formula implies that $f(u(x)) D_{p^{1 / \mu}, q^{1 / \mu}} u(x)$ is one of the $(p, q)$-antiderivatives of $f(x)$.

The $(p, q)$-integral is defined (see [22]) by

$$
\int f(x) d_{p, q} x=(p-q) \sum_{k=0}^{\infty} \frac{q^{k} x}{p^{k+1}} f\left(\frac{q^{k}}{p^{k+1}} x\right) .
$$


Let $f(x)=\sum_{k=0}^{\infty} a_{k} x^{k}$ be a formal power series. Applying $(p, q)$-integral to the both sides of $f(x)$ yields to

$$
\int f(x) d_{p, q} x=\sum_{k=0}^{\infty} a_{k} \frac{x^{k+1}}{[k+1]_{p, q}}+C
$$

where $C$ is a constant.

Some simple examples of $(p, q)$-integral are

$$
\begin{aligned}
\int(x \oplus y)_{p, q}^{n} d_{p, q} x & =\frac{\left(\frac{x}{p} \oplus y\right)_{p, q}^{n+1}}{[n+1]_{p, q}}+C, \\
\int \widetilde{e}_{p, q}(x) d_{p, q} x & =\widetilde{e}_{p, q}(x)+C, \\
\int e_{p, q}(x) d_{p, q} x & =e_{p, q}\left(\frac{x}{p}\right)+C, \\
\int E_{p, q}(x) d_{p, q} x & =E_{p, q}\left(\frac{x}{q}\right)+C .
\end{aligned}
$$

\section{3. $(p, q)$-Trigonometric Functions}

The $(p, q)$-analogues of the sine, cosine, tangent and cotangent functions can be defined in the same manner with their well known Euler expressions by means of the exponential functions.

Definition 2. Let $i=\sqrt{-1} \in \mathbb{C}$. Then two pairs of $(p, q)$-trigonometric functions are defined by

\begin{tabular}{|c|c|}
\hline $\sin _{p, q} x:=\frac{e_{p, q}(i x)-e_{p, q}(-i x)}{2 i}$, & $\operatorname{SIN}_{p, q} x:=\frac{E_{p, q}(i x)-E_{p, q}(-i x)}{2 i}$, \\
\hline $\cos _{p, q} x:=\frac{e_{p, q}(i x)+e_{p, q}(-i x)}{2}$, & $\operatorname{COS}_{p, q} x:=\frac{E_{p, q}(i x)+E_{p, q}(-i x)}{2}$, \\
\hline $\tan _{p, q} x:=\frac{\sin _{p, q} x}{\cos _{p, q} x}$, & $\operatorname{TAN}_{p, q} x:=\frac{\operatorname{SIN}_{p, q} x}{\operatorname{COS}_{p, q} x}$, \\
\hline $\cot _{p, q} x:=\frac{\cos _{p, q} x}{\sin _{p, q} x}$, & $\operatorname{COT}_{p, q} x:=\frac{\operatorname{COS}_{p, q} x}{\operatorname{SIN}_{p, q} x}$. \\
\hline
\end{tabular}

Using the identity (1.7), we have

$$
\sin _{p, q} x \operatorname{SIN}_{p, q} x=-\frac{e_{p, q}(i x) E_{p, q}(i x)+e_{p, q}(-i x) E_{p, q}(-i x)-2}{4}
$$

and

$$
\cos _{p, q} x \operatorname{COS}_{p, q} x=\frac{e_{p, q}(i x) E_{p, q}(i x)+e_{p, q}(-i x) E_{p, q}(-i x)+2}{4} .
$$

Thus, we derive the following formula

$$
\sin _{p, q} x \operatorname{SIN}_{p, q} x+\cos _{p, q} x \operatorname{COS}_{p, q} x=1,
$$

which is the $(p, q)$-analogue of the well-known formula

$$
\sin ^{2} x+\cos ^{2} x=1 \text {. }
$$


By means of the $(p, q)$-trigonometric functions, the corresponds secant and cosecant functions are described as

$$
\begin{array}{|c|c|}
\hline \sec _{p, q} x:=\frac{1}{\cos _{p, q} x}, & \csc _{p, q} x:=\frac{1}{\sin _{p, q} x}, \\
\hline \operatorname{SEC}_{p, q} x:=\frac{1}{\operatorname{COS}_{p, q} x}, & \operatorname{CSC}_{p, q} x:=\frac{1}{\operatorname{SIN}_{p, q} x} . \\
\hline
\end{array}
$$

Note that the $(p, q)$-tangent and $(p, q)$-cotangent functions coincide the following equalities

$$
\tan _{p, q} x=\mathrm{TAN}_{p, q} x \text { and } \cot _{p, q} x=\mathrm{COT}_{p, q} x,
$$

which are $(p, q)$-extension of the results in [6]. The two $(p, q)$-tangent functions are valid, however, one of these functions may be more useful than the other under special cases.

Now let us investigate $(p, q)$-derivatives of the $(p, q)$-trigonometric functions. By making use of (1.8), we readily see that

$$
\begin{aligned}
D_{p, q} \sin _{p, q} x & =D_{p, q}\left(\frac{e_{p, q}(i x)-e_{p, q}(-i x)}{2 i}\right) \\
& =\frac{D_{p, q} e_{p, q}(i x)-D_{p, q} e_{p, q}(-i x)}{2 i} \\
& =\frac{e_{p, q}(p x)+e_{p, q}(-p x)}{2}=\cos _{p, q}(p x) .
\end{aligned}
$$

By the same way, the others are stated as follows.

Theorem 2. The $(p, q)$-derivative operator fulfils the following equalities

\begin{tabular}{|c|c|}
\hline$D_{p, q} \sin _{p, q} x=\cos _{p, q}(p x)$, & $D_{p, q} \operatorname{SIN}_{p, q} x=\operatorname{COS}_{p, q}(q x)$, \\
\hline$D_{p, q} \cos _{p, q} x=-\sin _{p, q}(p x)$, & $D_{p, q} \operatorname{COS}_{p, q} x=-\operatorname{SIN}_{p, q}(q x)$, \\
\hline$D_{p, q} \tan _{p, q} x=1+\tan _{p, q}(p x) \tan _{p, q}(q x)$, & $D_{p, q} \operatorname{TAN}_{p, q} x=1+\operatorname{TAN}_{p, q}(p x) \operatorname{TAN}_{p, q}(q x)$, \\
\hline$D_{p, q} \cot _{p, q} x=-\frac{\sin _{p, q}^{2}(p x)+\cos _{p, q}^{2}(p x)}{\sin _{p, q}(p x) \sin _{p, q}(q x)}$, & $D_{p, q} \operatorname{COT}_{p, q} x=-\frac{\operatorname{SIN}_{p, q}^{2}(q x)+\operatorname{COS}_{p, q}^{2}(q x)}{\operatorname{SIN}_{p, q}(p x) \operatorname{SIN}_{p, q}(q x)}$, \\
\hline$D_{p, q} \sec _{p, q} x=\sec _{p, q}(q x) \tan _{p, q}(p x)$, & $D_{p, q} \operatorname{SEC}_{p, q} x=\operatorname{SEC}_{p, q}(p x) \operatorname{TAN}_{p, q}(q x)$, \\
\hline$D_{p, q} \csc _{p, q} x=-\csc _{p, q}(q x) \cot _{p, q}(p x)$, & $D_{p, q} \operatorname{CSC}_{p, q} x=-\mathrm{CSC}_{p, q}(p x) \operatorname{COT}_{p, q}(q x)$. \\
\hline
\end{tabular}

Now $(p, q)$-integration properties of the $(p, q)$-cosine and $(p, q)$-sine functions are given as follows.

Theorem 3. The following $(p, q)$-integrals are verified with easy computations:

\begin{tabular}{|c|}
\hline $\int \sin _{p, q} x d_{p, q} x=-\cos _{p, q}\left(\frac{x}{p}\right)+C$, \\
\hline $\int \cos _{p, q} x d_{p, q} x=\sin _{p, q}\left(\frac{x}{p}\right)+C$, \\
\hline $\int \operatorname{SIN}_{p, q} x d_{p, q} x=-\operatorname{COS}_{p, q}\left(\frac{x}{q}\right)+C$, \\
$\int \operatorname{COS}_{p, q} x d_{p, q} x=\operatorname{SIN}_{p, q}\left(\frac{x}{q}\right)+C$. \\
\hline
\end{tabular}


The $(p, q)$-exponential functions are related to the $(p, q)$-cosine and $(p, q)$-sine as

$$
\begin{aligned}
& e_{p, q}(i x)=\sum_{n=0}^{\infty} p^{\left(\begin{array}{c}
n \\
2
\end{array}\right) i^{n} \frac{x^{n}}{[n]_{p, q} !}} \\
& =\sum_{n=0}^{\infty} \frac{(-1)^{n}}{[2 n]_{p, q} !} p^{(2 n-1) n} x^{2 n}+i \sum_{n=0}^{\infty} \frac{(-1)^{n}}{[2 n+1]_{p, q} !} p^{(2 n+1) n} x^{2 n+1} \\
& =\cos _{p, q}(x)+i \sin _{p, q}(x)
\end{aligned}
$$

and

$$
\begin{aligned}
& E_{p, q}(i x)=\sum_{n=0}^{\infty} q^{\left(\begin{array}{c}
n \\
2
\end{array}\right)} i^{n} \frac{x^{n}}{[n]_{p, q} !} \\
& =\sum_{n=0}^{\infty} \frac{(-1)^{n}}{[2 n]_{p, q} !} q^{(2 n-1) n} x^{2 n}+i \sum_{n=0}^{\infty} \frac{(-1)^{n}}{[2 n+1]_{p, q} !} q^{(2 n+1) n} x^{2 n+1} \\
& =\operatorname{COS}_{p, q}(x)+i \operatorname{SIN}_{p, q}(x) \text {. }
\end{aligned}
$$

The following theorem includes a connection with $(p, q)$-sine and $(p, q)$-cosine functions.

Theorem 4. We have

$$
\sin _{p, q} x \operatorname{COS}_{p, q} x=\cos _{p, q} x \operatorname{SIN}_{p, q} x .
$$

The $(p, q)$-analogues of hyperbolic functions can be defined in the same manner with their well known Euler expressions by means of the exponential functions.

Definition 3. The $(p, q)$-hyperbolic functions are described as

\begin{tabular}{|c|c|}
\hline $\sinh _{p, q} x=\frac{e_{p, q}(x)-e_{p, q}(-x)}{2}$, & $\operatorname{SINH}_{p, q} x=\frac{E_{p, q}(x)-E_{p, q}(-x)}{2}$, \\
\hline $\cosh _{p, q} x=\frac{e_{p, q}(x)+e_{p, q}(-x)}{2}$, & $\mathrm{COSH}_{p, q} x=\frac{E_{p, q}(x)+E_{p, q}(-x)}{2}$, \\
\hline $\tanh _{p, q} x=\frac{\sinh _{p, q} x}{\cosh _{p, q} x}$, & $\operatorname{TANH}_{p, q} x=\frac{\operatorname{SINH}_{p, q} x}{\operatorname{COSH}_{p, q} x}$, \\
\hline $\operatorname{coth}_{p, q} x=\frac{\operatorname{Cosh}_{p, q} x}{\sinh _{p, q} x}$, & $\mathrm{COTH}_{p, q} x=\frac{\operatorname{COSH}_{p, q} x}{\operatorname{SINH}_{p, q} x}$. \\
\hline
\end{tabular}

The following relationhips hold true:

$$
\begin{array}{|l|l|}
\hline e_{p, q}(x)=\cosh _{p, q} x+\sinh _{p, q} x, & E_{p, q}(x)=\mathrm{COSH}_{p, q} x+\mathrm{SINH}_{p, q} x . \\
\hline
\end{array}
$$

Using the identity (1.7), we have

$$
\sinh _{p, q} x \operatorname{SINH}_{p, q} x=\frac{e_{p, q}(x) E_{p, q}(x)+e_{p, q}(-x) E_{p, q}(-x)-2}{4}
$$

and

$$
\cosh _{p, q} x \operatorname{COSH}_{p, q} x=\frac{e_{p, q}(x) E_{p, q}(x)+e_{p, q}(-x) E_{p, q}(-x)+2}{4} .
$$

Hence, we observe that

$$
\cosh _{p, q} x \mathrm{COSH}_{p, q} x-\sinh _{p, q} x \mathrm{SINH}_{p, q} x=1,
$$


which is the $(p, q)$-analogue of the well known formula

$$
\cosh ^{2} x-\sinh ^{2} x=1
$$

Following the $(p, q)$-hyperbolic functions, the hyperbolic $(p, q)$-secant and $(p, q)$-cosecant functions are defined by

\begin{tabular}{|l|l|}
\hline $\operatorname{sech}_{p, q} x:=\frac{1}{\cosh _{p, q} x}$, & $\operatorname{csch}_{p, q} x:=\frac{1}{\sinh _{p, q} x}$, \\
\hline $\operatorname{SECH}_{p, q} x:=\frac{1}{\operatorname{COSH}_{p, q} x}$, & $\operatorname{CSCH}_{p, q} x:=\frac{1}{\operatorname{SINH}_{p, q} x}$. \\
\hline
\end{tabular}

The following theorem consist of the $(p, q)$-derivative properties of $(p, q)$-hyperbolic functions.

Theorem 5. The $(p, q)$-derivative operator fulfils the following equations

\begin{tabular}{|c|c|}
\hline$D_{p, q} \sinh _{p, q} x=\cosh _{p, q}(p x)$, & $D_{p, q} \operatorname{SINH}_{p, q} x=\operatorname{COSH}_{p, q}(q x)$, \\
\hline$D_{p, q} \cosh _{p, q} x=\sinh _{p, q}(p x)$, & $D_{p, q} \operatorname{COSH}_{p, q} x=\operatorname{SINH}_{p, q}(q x)$, \\
\hline$D_{p, q} \tanh _{p, q} x=1-\tanh _{p, q}(p x) \tanh _{p, q}(q x)$, & $D_{p, q} \operatorname{TANH}_{p, q} x=1-\operatorname{TANH}_{p, q}(p x) \operatorname{TANH}_{p, q}(q x)$, \\
\hline$D_{p, q} \operatorname{coth}_{p, q} x=\frac{\sinh _{p, q}^{2}(p x)-\cosh _{p, q}^{2}(p x)}{\sinh _{p, q}(p x) \sinh _{p, q}(q x)}$, & $D_{p, q} \operatorname{COTH}_{p, q} x=\frac{\operatorname{SINH}_{p, q}(q x)-\mathrm{COSH}_{p, q}^{2}(q x)}{\operatorname{SINH}_{p, q}(p x) \operatorname{SINH}_{p, q}(q x)}$, \\
\hline$D_{p, q} \operatorname{sech}_{p, q} x=-\operatorname{sech}_{p, q}(q x) \tanh _{p, q}(p x)$, & $D_{p, q} \operatorname{SECH}_{p, q} x=-\operatorname{SECH}_{p, q}(p x) \operatorname{TANH}_{p, q}(q x)$, \\
\hline$D_{p, q} \operatorname{csch}_{p, q} x=-\operatorname{csch}_{p, q}(q x) \operatorname{coth}_{p, q}(p x)$, & $D_{p, q} \mathrm{CSCH}_{p, q} x=-\mathrm{CSCH}_{p, q}(p x) \mathrm{COTH}_{p, q}(q x)$. \\
\hline
\end{tabular}

The following theorem includes the $(p, q)$-integral properties of $(p, q)$-hyperbolic functions.

Theorem 6. We have

\begin{tabular}{|c|}
\hline $\int \sinh _{p, q}(x) d_{p, q} x=\cosh _{p, q}\left(\frac{x}{p}\right)+C$, \\
\hline $\int \cosh _{p, q}(x) d_{p, q} x=\sinh _{p, q}\left(\frac{x}{p}\right)+C$, \\
\hline $\int \operatorname{SINH}_{p, q}(x) d_{p, q} x=\operatorname{COSH}_{p, q}\left(\frac{x}{q}\right)+C$, \\
\hline $\int \operatorname{COSH}_{p, q}(x) d_{p, q} x=\operatorname{SINH}_{p, q}\left(\frac{x}{q}\right)+C$. \\
\hline
\end{tabular}

The $(p, q)$-exponential functions are related to the $(p, q)$ - hyperbolic cosine and $(p, q)$ hyperolic sine by

$$
\begin{aligned}
& e_{p, q}(x)=\sum_{n=0}^{\infty} p^{\left(\begin{array}{l}
n \\
2
\end{array}\right)} \frac{x^{n}}{[n]_{p, q} !} \\
& =\sum_{n=0}^{\infty} \frac{(-1)^{n}}{[2 n]_{p, q} !} p^{(2 n-1) n} x^{2 n}+\sum_{n=0}^{\infty} \frac{(-1)^{n}}{[2 n+1]_{p, q} !} p^{(2 n+1) n} x^{2 n+1} \\
& =\cosh _{p, q}(x)+\sinh _{p, q}(x)
\end{aligned}
$$


and

$$
\begin{aligned}
E_{p, q}(x) & =\sum_{n=0}^{\infty} q^{\left(\begin{array}{c}
n \\
2
\end{array}\right) \frac{x^{n}}{[n]_{p, q} !}} \\
& =\sum_{n=0}^{\infty} \frac{(-1)^{n}}{[2 n]_{p, q} !} q^{(2 n-1) n} x^{2 n}+\sum_{n=0}^{\infty} \frac{(-1)^{n}}{[2 n+1]_{p, q} !} q^{(2 n+1) n} x^{2 n+1} \\
& =\operatorname{COSH}_{p, q} x+\operatorname{SINH}_{p, q} x
\end{aligned}
$$

\begin{tabular}{|c|c|}
\hline $\sin _{\frac{1}{p}, \frac{1}{q}} x=\operatorname{SIN}_{p, q} x$ & $\operatorname{SIN}_{\frac{1}{p}, \frac{1}{q}} x=\sin _{p, q} x$ \\
\hline $\cos _{\frac{1}{p}, \frac{1}{q}} x=\operatorname{COS}_{p, q} x$, & $\operatorname{COS}_{\frac{1}{p}, \frac{1}{q}} x=\cos _{p, q} x$, \\
\hline $\tan _{\frac{1}{p}, \frac{1}{q}} x=\mathrm{TAN}_{p, q} x$ & $\operatorname{TAN}_{\frac{1}{p}, \frac{1}{q}} x=\tan _{p, q} x$ \\
\hline $\cot _{\frac{1}{p}, \frac{1}{q}} x=\mathrm{COT}_{p, q} x$, & $\operatorname{COT}_{\frac{1}{p}, \frac{1}{q}} x=\cot _{p, q} x$ \\
\hline $\sec _{\frac{1}{p}, \frac{1}{q}} x=\mathrm{SEC}_{p, q} x$ & $\operatorname{SEC}_{\frac{1}{p}, \frac{1}{q}} x=\sec _{p, q} x$ \\
\hline $\csc _{\frac{1}{p}, \frac{1}{q}} x=\mathrm{CSC}_{p, q} x$ & $\mathrm{CSC}_{\frac{1}{p}, \frac{1}{q}} x=\csc _{p, q} x$ \\
\hline $\sinh _{\frac{1}{p}, \frac{1}{q}} x=\operatorname{SINH}_{p, q} x$, & $\operatorname{SINH}_{\frac{1}{p}, \frac{1}{q}} x=\sinh _{p, q} x$ \\
\hline $\cosh _{\frac{1}{p}, \frac{1}{q}} x=\mathrm{COSH}_{p, q} x$, & $\mathrm{COSH}_{\frac{1}{p}, \frac{1}{q}} x=\cosh _{p, q} x$, \\
\hline $\tanh _{\frac{1}{p}, \frac{1}{q}} x=\mathrm{TANH}_{p, q} x$, & $\mathrm{TANH}_{\frac{1}{p}, \frac{1}{q}} x=\tanh _{p, q} x$, \\
\hline $\operatorname{coth}_{\frac{1}{p}, \frac{1}{q}} x=\mathrm{COTH}_{p, q} x$, & $\mathrm{COTH}_{\frac{1}{p}, \frac{1}{q}} x=\operatorname{coth}_{p, q} x$, \\
\hline $\operatorname{sech}_{\frac{1}{p}, \frac{1}{q}} x=\mathrm{SECH}_{p, q} x$, & $\mathrm{SECH}_{\frac{1}{p}, \frac{1}{q}} x=\operatorname{sech}_{p, q} x$, \\
\hline $\operatorname{csch}_{\frac{1}{p}, \frac{1}{q}} x=\mathrm{CSCH}_{p, q} x$, & $\mathrm{CSCH}_{\frac{1}{p}, \frac{1}{q}} x=\operatorname{csch}_{p, q} x$. \\
\hline
\end{tabular}

Using the formulas (2.5) and (2.6), we obtain the following results.

Theorem 7. The following identities are readily attested:

In terms of the new $(p, q)$-exponential function $\widetilde{e}_{p, q} x$, the corresponds trigonometric functions can be defined as

\begin{tabular}{|c|c|}
\hline$\widetilde{\sin }_{p, q} x:=\frac{\widetilde{e}_{p, q}(i x)-\widetilde{e}_{p, q}(-i x)}{2 i}$, & $\widetilde{\tan }_{p, q} x:=\frac{\widetilde{\sin }_{p, q} x}{\widetilde{\cos }_{p, q} x}$, \\
\hline$\widetilde{\cos }_{p, q} x:=\frac{\widetilde{e}_{p, q}(i x)+\widetilde{e}_{p, q}(-i x)}{2}$, & $\widetilde{\cot } p, q x:=\frac{\widetilde{\cos }_{p, q} x}{\widetilde{\sin }_{p, q} x}$, \\
\hline$\widetilde{\sec }_{p, q} x=\frac{1}{\widetilde{\cos }_{p, q} x}$, & $\widetilde{\csc }_{p, q} x=\frac{1}{\widetilde{\sin }_{p, q} x}$, \\
\hline
\end{tabular}


which satisfy

\begin{tabular}{|c|}
\hline$D_{p, q} \widetilde{\sin }_{p, q} x=\widetilde{\cos }_{p, q} x$ \\
\hline$D_{p, q} \widetilde{\cos }_{p, q} x=-\widetilde{\sin }_{p, q} x$ \\
\hline$D_{p, q} \widetilde{\tan }_{p, q} x=\frac{\widetilde{\cos }_{p, q} x+\widetilde{\tan }_{p, q}(p x) \sin _{p, q} x}{\widetilde{\cos }_{p, q}(q x)}$, \\
\hline$D_{p, q} \widetilde{\cot }_{p, q} x=-\frac{\widetilde{\sin }_{p, q} x+\widetilde{\cot }_{p, q}(p x) \widetilde{\cos }_{p, q} x}{\widetilde{\sin }_{p, q}(q x)}$, \\
\hline$D_{p, q} \widetilde{\sec }_{p, q} x=\frac{\sin _{p, q} x}{\widetilde{\cos }_{p, q}(p x) \widetilde{\cos }_{p, q}(q x)}$, \\
\hline$D_{p, q} \widetilde{\csc }_{p, q} x=-\frac{\widetilde{\cos }_{p, q} x}{\widetilde{\sin }_{p, q}(p x) \widetilde{\sin }_{p, q}(q x)}$. \\
\hline
\end{tabular}

The $(p, q)$-exponential function $\widetilde{e}_{p, q} x$ are correlated with the new $(p, q)$-cosine and $(p, q)$ sine functions as

$$
\begin{aligned}
\widetilde{e}_{p, q}(i x) & =\sum_{n=0}^{\infty} i^{n} \frac{x^{n}}{[n]_{p, q} !} \\
& =\sum_{n=0}^{\infty} \frac{(-1)^{n}}{[2 n]_{p, q} !} x^{2 n}+i \sum_{n=0}^{\infty} \frac{(-1)^{n}}{[2 n+1]_{p, q} !} x^{2 n+1} \\
& =\widetilde{\cos }_{p, q}(x)+i \widetilde{\sin }_{p, q}(x) .
\end{aligned}
$$

\begin{tabular}{|c|c|}
\hline$\widetilde{\sinh }_{p, q} x:=\frac{\widetilde{e}_{p, q}(x)-\widetilde{e}_{p, q}(-x)}{2}$ & $\widetilde{\tanh }_{p, q} x:=\frac{\sinh _{p, q} x}{\widetilde{\cosh }_{p, q} x}$ \\
\hline$\widetilde{\cosh }_{p, q} x:=\frac{\widetilde{e}_{p, q}(x)+\widetilde{e}_{p, q}(-x)}{2}$ & $\widetilde{\operatorname{coth}}_{p, q} x:=\frac{\widetilde{\cosh }_{p, q} x}{\widetilde{\sinh }_{p, q} x}$ \\
\hline$\widetilde{\operatorname{sech}}_{p, q} x=\frac{1}{\widetilde{\cosh _{p, q} x}}$ & $\widetilde{\operatorname{csch}}_{p, q} x=\frac{1}{\widetilde{\sinh _{p, q} x}}$ \\
\hline
\end{tabular}

In terms of the new $(p, q)$-exponential fuction $\widetilde{e}_{p, q} x$, the correspond hyperbolic functions are described as 
which fulfil

\begin{tabular}{|c|}
\hline$D_{p, q} \widetilde{\sinh }_{p, q} x=\widetilde{\cosh }_{p, q} x$ \\
\hline$D_{p, q} \widehat{\cosh }_{p, q} x=\widehat{\sinh }_{p, q} x$ \\
\hline$D_{p, q} \widetilde{\tanh }_{p, q} x=\frac{\widetilde{\cosh }_{p, q} x+\widetilde{\tanh }_{p, q}(p x) \widetilde{\sinh }_{p, q} x}{\widetilde{\cosh }_{p, q}(q x)}$ \\
\hline$D_{p, q} \widetilde{\operatorname{coth}}_{p, q} x=-\frac{\widetilde{\sinh }_{p, q} x-\widetilde{\operatorname{coth}}_{p, q}(p x) \widetilde{\cosh }_{p, q} x}{\widetilde{\sinh }_{p, q}(q x)}$, \\
\hline$D_{p, q} \widetilde{\operatorname{sech}}_{p, q} x=-\frac{\widetilde{\sinh }_{p, q} x}{\widetilde{\cosh }_{p, q}(p x) \widetilde{\cosh }_{p, q}(q x)}$, \\
\hline$D_{p, q} \widetilde{\operatorname{csch}}_{p, q} x=-\frac{\widetilde{\cosh }_{p, q} x}{\widetilde{\sinh }_{p, q}(p x) \widetilde{\sinh }_{p, q}(q x)}$. \\
\hline
\end{tabular}

Theorem 8. The following $(p, q)$-integrals are valid:

$$
\begin{array}{|l|}
\hline \int \widetilde{\sin }_{p, q} x d_{p, q} x=-\widetilde{\cos }_{p, q} x+C, \\
\int \widetilde{\cos }_{p, q} x d_{p, q} x=\widetilde{\sin }_{p, q} x+C, \\
\int \widetilde{\sinh }_{p, q} x d_{p, q} x=\widetilde{\cosh }_{p, q} x+C, \\
\int \widetilde{\cosh }_{p, q} x d_{p, q} x=\widetilde{\sinh }_{p, q} x+C . \\
\hline
\end{array}
$$

New $(p, q)$-trigonometric functions can be expressed by earlier defined $(p, q)$-trigonometric functions as follows.

Theorem 9. The following equalities

\begin{tabular}{|c|}
\hline$\widetilde{\sin }_{p, q}(x \oplus x)_{p, q}=\sin _{p, q} x \operatorname{COS}_{p, q} x+\cos _{p, q} x \operatorname{SIN}_{p, q} x$, \\
\hline$\widetilde{\sin }_{p, q}(x \ominus x)_{p, q}=\sin _{p, q} x \operatorname{COS}_{p, q} x-\cos _{p, q} x \operatorname{SIN}_{p, q} x$, \\
\hline$\widetilde{\cos }_{p, q}(x \oplus x)_{p, q}=\cos _{p, q} x \operatorname{COS}_{p, q} x-\sin _{p, q} x \operatorname{SIN}_{p, q} x$, \\
\hline$\widetilde{\cos }_{p, q}(x \ominus x)_{p, q}=\cos _{p, q} x \operatorname{COS}_{p, q} x+\sin _{p, q} x \operatorname{SIN}_{p, q} x$, \\
\hline $\sinh _{p, q}(x \oplus x)_{p, q}=\sinh _{p, q} x \operatorname{COSH}_{p, q} x+\cosh _{p, q} x \operatorname{SINH}_{p, q} x$, \\
\hline $\sinh _{p, q}(x \ominus x)_{p, q}=\sinh _{p, q} x \operatorname{COSH}_{p, q} x-\cosh _{p, q} x \operatorname{SINH}_{p, q} x$, \\
\hline $\cosh _{p, q}(x \oplus x)_{p, q}=\cosh _{p, q} x \operatorname{COSH}_{p, q} x+\sinh _{p, q} x \operatorname{SINH}_{p, q} x$, \\
\hline $\cosh _{p, q}(x \ominus x)_{p, q}=\cosh _{p, q} x \operatorname{COSH}_{p, q} x-\sinh _{p, q} x \operatorname{SINH}_{p, q} x$ \\
\hline
\end{tabular}

are true.

The following intriguing identities between $(p, q)$-trigonometric and $(p, q)$-hyperbolic functions hold true. 
Theorem 10. We have

\begin{tabular}{|c|c|c|}
\hline $\sinh _{p, q} x=-i \sin _{p, q}(i x)$ & & \\
$\operatorname{SINH}_{p, q} x=-i \operatorname{SIN}_{p, q}(i x)$ & $q \rightarrow p=1$ & $\sinh x=-i \sin (i x)$, \\
$\widetilde{\sinh }_{p, q} x=-i \widetilde{\sin }_{p, q}(i x)$ & & \\
\hline $\cosh _{p, q} x=\cos _{p, q}(i x)$ & & $\cosh x=\cos (i x)$, \\
$\operatorname{COSH}_{p, q} x=\operatorname{COS}_{p, q}(i x)$ & $q \rightarrow p=1$ & \\
$\widetilde{\cosh }_{p, q} x=\widetilde{\cos }_{p, q}(i x)$ & & $\tanh x=-i \tan (i x)$, \\
$\tanh _{p, q} x=-i \tan _{p, q}(i x)$ & & \\
$\operatorname{TANH}_{p, q} x=-i \operatorname{TAN}_{p, q}(i x)$ & $q \rightarrow p=1$ & \\
$\tanh _{p, q} x=-i \tan _{p, q}(i x)$ & & $\operatorname{coth} x=i \cot (i x)$. \\
\hline $\operatorname{coth}_{p, q} x=i \cot _{p, q}(i x)$ & & \\
$\operatorname{COTH}_{p, q} x=i \operatorname{COT}_{p, q}(i x)$ & $q \rightarrow p=1$ & \\
$\operatorname{coth}_{p, q} x=i \cot _{p, q}(i x)$ & &
\end{tabular}

Here the $(p, q)$-trigonometric functions and the $(p, q)$-hypergeometric functions are examined whether these functions are odd functions or even functions.

Theorem 11. We have

\begin{tabular}{|c|c|c|}
\hline $\sin _{p, q}(-x)=-\sin _{p, q} x$ & $\operatorname{SIN}_{p, q}(-x)=-\operatorname{SIN}_{p, q} x$, & $\underbrace{}_{\sin _{p, q}(-x)=-\sin _{p, q} x,}$ \\
\hline $\cos _{p, q}(-x)=\cos _{p, q} x$ & $\mathrm{COS}_{p, q}(-x)=\mathrm{COS}_{p, q} x$ & $\widetilde{\cos }_{p, q}(-x)=\widetilde{\cos }_{p, q} x$ \\
\hline $\tan _{p, q}(-x)=-\tan _{p, q} x$ & $\operatorname{TAN}_{p, q}(-x)=-\mathrm{TAN}_{p, q} x$ & $\tan _{p, q}(-x)=-\tan _{p, q} x$ \\
\hline $\cot _{p, q}(-x)=-\cot _{p, q} x$ & $\mathrm{COT}_{p, q}(-x)=-\mathrm{COT}_{p, q} x$, & $\widetilde{\cot }_{p, q}(-x)=-\widetilde{\cot }_{p, q} x$ \\
\hline $\sec _{p, q}(-x)=\sec _{p, q} x$ & $\mathrm{SEC}_{p, q}(-x)=\mathrm{SEC}_{p, q} x$ & $\widetilde{\sec }_{p, q}(-x)=\widetilde{\sec }_{p, q} x$ \\
\hline $\csc _{p, q}(-x)=-\csc _{p, q} x$ & $\mathrm{CSC}_{p, q}(-x)=-\mathrm{CSC}_{p, q} x$ & $\widetilde{\csc }_{p, q}(-x)=-\widetilde{\csc }_{p, q} x$ \\
\hline $\sinh _{p, q}(-x)=-\sinh _{p, q} x$, & $\mathrm{SINH}_{p, q}(-x)=-\mathrm{SINH}_{p, q} x$ & $\sinh _{p, q}(-x)=-\sinh _{p, q} x$, \\
\hline $\cosh _{p, q}(-x)=\cosh _{p, q} x$ & $\mathrm{COSH}_{p, q}(-x)=\mathrm{COSH}_{p, q} x$ & $\cosh _{p, q}(-x)=\cosh _{p, q} x$ \\
\hline $\tanh _{p, q}(-x)=-\tanh _{p, q} x$ & $\operatorname{TANH}_{p, q}(-x)=-\mathrm{TANH}_{p, q} x$ & $\tanh _{p, q}(-x)=-\tanh _{p, q} x$ \\
\hline $\operatorname{coth}_{p, q}(-x)=-\operatorname{coth}_{p, q} x$ & $\mathrm{COTH}_{p, q}(-x)=-\mathrm{COTH}_{p, q} x$, & $\operatorname{coth}_{p, q}(-x)=-\operatorname{coth}_{p, q} x$, \\
\hline $\operatorname{sech}_{p, q}(-x)=\operatorname{sech}_{p, q} x$ & $\mathrm{SECH}_{p, q}(-x)=\mathrm{SECH}_{p, q} x$ & $\operatorname{sech}_{p, q}(-x)=\operatorname{sech}_{p, q} x$ \\
\hline $\operatorname{csch}_{p, q}(-x)=-\operatorname{csch}_{p, q} x$, & $\mathrm{CSCH}_{p, q}(-x)=-\mathrm{CSCH}_{p, q} x$, & $\operatorname{csch}_{p, q}(-x)=-\operatorname{csch}_{p, q} x$. \\
\hline
\end{tabular}

\section{References}

[1] M. Arik, E. Demircan, T. Turgut, L. Ekinci, M. Mungan, Fibonacci oscillators, Z. Phys. C: Particles and Fields, 55 (1991), 89-95.

[2] G. Brodimas, A. Jannussis, R. Mignani, Two-parameter quantum groups, Universita di Roma Preprint Nr. 820 (1991).

[3] M. Burban, A. U. Klimyk, $P, Q$-differentiation, $P, Q$-integration and $P, Q$-hypergeometric functions related to quantum groups, Integral Transforms Spec. Funct., 2 (1994), 15-36.

[4] R. Chakrabarti and R. Jagannathan, $A(p, q)$-oscillator realization of two-parameter quantum algebras, J. Phys. A: Math. Gen., 24 (1991), L711.

[5] G. S. Cheon, A note on the Bernoulli and Euler polynomials, Appl. Math. Lett., 16 (2003) 365-368.

[6] J. L. Cieśliński, Improved q-exponential and q-trigonometric functions, arXiv:1006.5652v1 [math.CA] (2010).

[7] R. B. Corcino, On P, Q-Binomial coefficients, Electron. J. Combin. Number Theory, 8 (2008), \#A29. 
[8] U. Duran, M. Acikgoz, S. Araci, $(p, q)$-Volkenborn integral, submitted to J. Number Theory.

[9] U. Duran, M. Acikgoz, S. Araci, On some polynomials derived from $(p, q)$-calculus, accepted in J. Comput. Theor. Nanosci. (2016).

[10] U. Duran, M. Acikgoz, S. Araci, Research on some new results arising from multiple q-calculus, accepted in Filomat (2016).

[11] U. Duran, M. Acikgoz, S. Araci, H. M. Srivastava, A certain $(p, q)$-derivative operator and associated divided differences, accepted in J. Comput. Theor. Nanosci. (2016).

[12] U. Duran, M. Acikgoz, S. Araci, On ( $p, q)$-Bernoulli, $(p, q)$-Euler and ( $p, q)$-Genocchi polynomials, accepted in J. Comput. Theor. Nanosci. (2016).

[13] T. Ernst, The history of q-calculus and a new method, U.U.D.M. Department of Mathematics, Uppsala University, Uppsala (2000) Report 2000, 16.

[14] F. H. Jackson, On q-definite integrals. Pure Appl. Math. Q. 41, 193-203 (1910).

[15] R. Jagannathan, K. S. Rao, Two-parameter quantum algebras, twin-basic numbers, and associated generalized hypergeometric series, in Proceeding of the International Conference on Number Theory and Mathematical Physics, Srinivasa Ramanujan Centre, Kumbakonam, India, 20-21 December 2005.

[16] R. Jagannathan, (P,Q)-Special functions, Special Functions and Differential Equations, Proceedings of a Workshop held at The Institute of Mathematical Sciences, Matras, India, January (1997), 13-24.

[17] V. Kac and P. Cheung, Quantum calculus, New York: Springer, (2002).

[18] T. Kim, q-extension of the Euler formula and trigonometric functions, Russ. J. Math. Phys., vol. 14 (2007), no. 3, pp. 275-278.

[19] M. Mursaleen, K. J. Ansari, A. Khan, On $(p, q)$-analogue of Bernstein operators, Appl. Math. Comput., Volume 266 (2015) 874-882.

[20] M. Mursaleen, K. J. Ansari, A. Khan, Erratum to "On ( $p, q)$-analogue of Bernstein operators" [Appl. Math. Comput. 266 (2015) 874-882]., Appl. Math. Comput. Volume 278 (2016) 70-71.

[21] S. Nalci, O. K. Nalci, Exactly solvable q-extended nonlinear classical and quantum models, Lambert Academic Publishing, 2014.

[22] P. N. Sadjang, On the fundamental theorem of $(p, q)$-calculus and some $(p, q)$-Taylor formulas, arXiv:1309.3934 [math.QA] (2013).

[23] P. N. Sadjang, On the $(p, q)$-Gamma and the $(p, q)$-Beta functions, arXiv 1506.07394v1 [math.QA], (2015).

[24] H. M. Srivastava, A. Pinter, Remarks on Some Relationships Between the Bernoulli and Euler Polynomails, Appl. Math. Lett., 17 (2004), 375-380.

[25] M. Wachs, D. White, p, q-Stirling numbers and set partition statistics, J. Conbin. Theory, A 56 (1991), $27-46$. 